\title{
Status of the Development of X-Band Antenna-Amplifier: Design, Simulations, and Prototype Experiments ${ }^{1}$
}

\author{
A.S. Shlapakovski, S.N. Artemenko, V.A. Avgustinovich, A.I. Mashchenko, \\ V.M. Matvienko, V.Yu. Mityushkina, I.I. Vintizenko, W. Jiang*, and E. Schamiloglu** \\ Nuclear Physics Institute, 2 a Lenina Str., Tomsk, 634050, Russia \\ Phone: 7-382-241-7959,Fax: 7-382-242-3934,E-mail: shl@npi.tpu.ru \\ * Nagaoka University of Technology, Nagaoka, Niigata 940-2188, Japan \\ ** University of New Mexico, Albuquerque NM, 87131, USA
}

\begin{abstract}
New results obtained in the course of the development of the $\mathrm{X}$-band antenna-amplifier, potentially controllable high-power microwave source driven by the compact module of linear induction accelerator (LIA), are reported. The design of the device including a microwave pulse compressor as RF drive source is presented. Numerical simulations using the 3 -D version of the PIC code MAGIC show that the output power of $\sim 30 \mathrm{MW}$ is achievable with $\sim 290 \mathrm{kV}$ operating voltage and $\sim 1.17 \mathrm{kA}$ beam current at $\sim 9.3 \mathrm{GHz}$ frequency. The prototype experiments included the annular electron beam transport with a dielectric rod inside (no input microwave signal) and low-power microwave transmission in the absence of the beam. The possibility to avoid plasma formation at the rod surface to the inadmissible degree (when the density much exceeds $10^{12} \mathrm{~cm}^{-3}$ ) has been demonstrated; thus, the concept of the antenna-amplifier can be considered realizable. The measurements with the microwave transmission system incorporated into the LIA module show that the real geometry practically does not change the rod antenna characteristics in comparison with the case of isolated antenna.
\end{abstract}

\section{Introduction}

A hybrid antenna-amplifier is a relativistic TWT with a dielectric rod-based slow wave structure in which an annular electron beam amplifies the fundamental, nonaxisymmetric $\mathrm{HE}_{11}$ mode of the dielectric rod antenna. The concept of the antenna-amplifier was described earlier in detail $[1,2]$. Its key feature is that the annular beam is generated in the LIA module in which a high-voltage electrode is at ground potential from the external side, i.e., can be directly connected to an external microwave source. As a result, the hollow cathode holder can play the role of the rod antenna feed waveguide, and the antenna feed signal serves as the TWT RF drive. Such device seems promising as a compact controllable high-power X-band source.
Our work on the antenna-amplifier development is aimed at the proof-of-principle gain demonstration experiment. It is planned to check the range of possibilities for the control of output radiation parameters in the single-shot mode of LIA operation. The work includes, on the one hand, designing all details of the future experiment and carrying out numerical simulations predicting its expected results and, on the other hand, conducting model experiments on beam transport and no-beam low-power microwave transmission. The goal of beam transport experiments is to estimate the density of plasma that can appear at the surface of the dielectric rod located within the hollow cathode and annular beam, since too dense plasma could prevent a Cherenkov interaction of the beam with the $\mathrm{HE}_{11}$ mode. For the low-power microwave "cold" experiments, the main objective is to determine the influence of real surrounding geometry on the level of reflections and directivity of the dielectric rod antenna.

\section{Design of the proof-of-principle experiment}

As was shown earlier $[1,2]$, depending on the parameters of electron beam and dielectric rod in the interaction space, there can be two different physical situations in the antenna-amplifier with regard to the $\mathrm{HE}_{11}$ mode prevalence. For initial proof-of-principle experiments, we set aside the configuration allowing for the RF drive frequency harmonics generation [2] and have chosen the set of parameters corresponding to the situation of the $\mathrm{HE}_{11}$ mode being dominant.

So, the designed parameters of the interaction region are as follows. The diameters of the drift tube and dielectric rod are $40 \mathrm{~mm}$ and $12 \mathrm{~mm}$, respectively. The dielectric constant of the rod material $\varepsilon=5$. The gap between the electron beam and the dielectric surface is $4 \mathrm{~mm}$. The length of the interaction space is to be varied within the range of 20 to $30 \mathrm{~cm}$ in order to find the optimal length giving maximum gain of the antenna-amplifier in the nonlinear regime. To provide this variation, it is only needed to change the length of the uniform region of guiding magnetic field; this is

\footnotetext{
${ }^{1}$ The work was supported in part by Russian Foundation for Basic Research (grant no. 05-08-01150) and Asian Office of Aerospace Research and Development (AOARD-05-4041).
} 
an additional advantage compared to conventional relativistic TWTs where a physical length of a slowwave structure should be changed too. The length of the solenoid to be used is $60 \mathrm{~cm}$, and the magnetic field at the axis is up to $3.6 \mathrm{~T}$ with the available battery. The interaction region length can be varied by two different means: moving the solenoid along the system axis or using a movable copper ring inside it at the output end.

The LIA module is based on the multi-channel spark gap switching that provides the discharge of strip forming lines through magnetization turns of the induction system. In detail, the LIA design was described in [2]. The module is relatively compact $(\sim 70$ $\mathrm{cm}$ diameter and $\sim 100 \mathrm{~cm}$ length along with the power supply). The accelerating voltage can be varied from 250 to $340 \mathrm{kV}$ corresponding to the electron beam current of 0.9 to $1.5 \mathrm{kA}$ (for the diode geometry used in the experiments on beam transport). The voltage pulse duration is $\sim 120 \mathrm{~ns}$.

In the experiments on beam transport, the beam was produced from the coaxial magnetically insulated diode with $60 \mathrm{~mm}$ anode and $20 \mathrm{~mm}$ cathode diameters. For the proof-of-principle experiments, the anode has been modified to get the diode of quasi-planar geometry that allows for beam current variation at a given accelerating voltage and shortens the length of electrons acceleration to the energy they acquire in the interaction region.

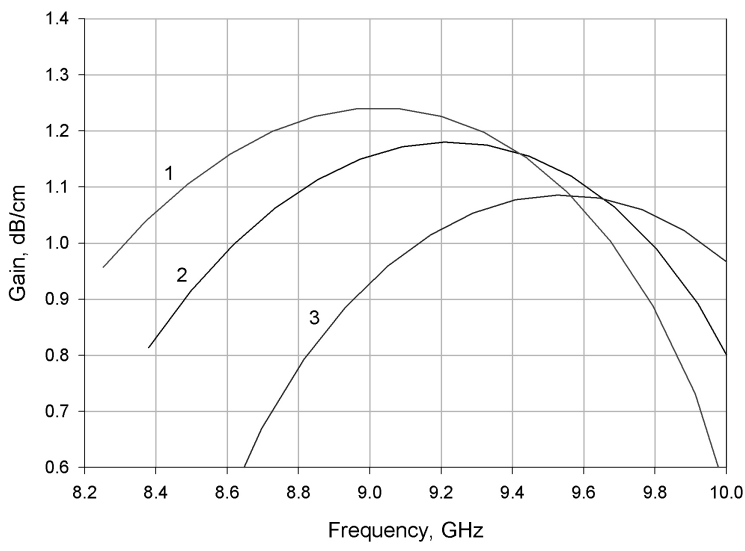

Fig. 1. Antenna-amplifier gain calculated from linear theory for the design parameters at different anode voltages $U$ determining beam current $I_{b}$ and Lorentz factor $\gamma$ in the interaction space.

$1-\gamma=1.51, I_{b}=1.5 \mathrm{kA}(U=343 \mathrm{kV})$;

$2-\gamma=1.48, I_{b}=1.36 \mathrm{kA}(U=322 \mathrm{kV})$;

$3-\gamma=1.44, I_{b}=1.19 \mathrm{kA}(U=294 \mathrm{kV})$.

The gain vs. frequency dependences calculated for the $\mathrm{HE}_{11}$ mode from the dispersion relation of the linear theory [1] at the interaction space design parameters and three sets of beam current and electrons Lorentz factor (corresponding to different anode voltages from within the range of LIA operation at the given diode geometry) are presented in Fig. 1. It is seen from this figure that the proper choice for the RF drive frequency is from within the range of 9.0 to $9.5 \mathrm{GHz}$. This agrees with the tuning range of the available magnetron to be employed for the RF drive pulse generation (8.6-9.6 GHz at the output power level of 100 to $200 \mathrm{~kW})$.

An important feature of the design is that the source of the RF drive signal is supposed to be the microwave pulse compressor. Using the pulse compression technique provides the RF drive pulse length shorter than the beam pulse length. This is typical for classical TWT amplifiers and does not take place, as yet, in relativistic ones, in which microsecond pulsed magnetrons are usually employed, whereas pulse durations of high-current accelerators are of 10-100 ns. This makes impossible the phase control of the output signal due to an uncontrollable phase shift during a voltage pulse rise-time. With the microwave pulse compressor, the beam parameters would be stable during the RF drive pulse, and also, this allows one to increase the input microwave power up to megawatt level.

For experiments on gain demonstration in the antenna-amplifier, the special X-band pulse compressor has been designed. Its expected output pulse duration is $\sim 4 \mathrm{~ns}$ FWHM at the peak power of $\sim 0.5 \mathrm{MW}$ and above, up to several MWs. So high peak power is achievable since the compressor design is based on the overmoded cylindrical storage cavity, and lower power level is obtained by decreasing the value of input coupling (size of the cavity coupling hole) down from the optimum. In order to demonstrate the control of the antenna-amplifier radiation frequency, the compressor is designed for two operating frequencies (9.1 and $9.4 \mathrm{GHz}$ ); one can switch over the frequency by tuning the magnetron and switching over the cavity operating modes, $\mathrm{TE}_{11(27)}$ and $\mathrm{TE}_{11(28)}$, respectively. The length of the storage cavity along with the output tee is $\sim 57 \mathrm{~cm}$.

The microwave transmission system from the compressor output to the antenna-amplifier interaction space is incorporated into the LIA module, so that the key feature of the device concept is realized, the antenna feed waveguide could serve as the LIA cathode holder. This system has been designed, fabricated, and tested; its schematic is shown in Fig. 2. Inside the LIA center electrode 7 , the standard X-band rectangular waveguide 1 and the buffer section 11 representing a rectangular-to-circular waveguide transformer are placed. At the external, grounded side, the flange 2 and fixing nut 5 provide the contact with the center electrode and the end flange of LIA tank 9. The flange 2 is connected to the standard ninety-degree bend, so that the compressor (not shown) is placed sideways. At the opposite end, the choke waveguide flange 3 with the vacuum window 4 is fixed by means of the 
coupling nut 6 at the buffer section 11, which is, in its turn, fixed at the LIA high-voltage flange 12 from the vacuum side. The cathode holder 14 is connected to the buffer section. The inner surface of the cathode holder represents a waveguide transformer to the end waveguide exciting the $\mathrm{HE}_{11}$ mode of the dielectric rod through the horn (cathode).

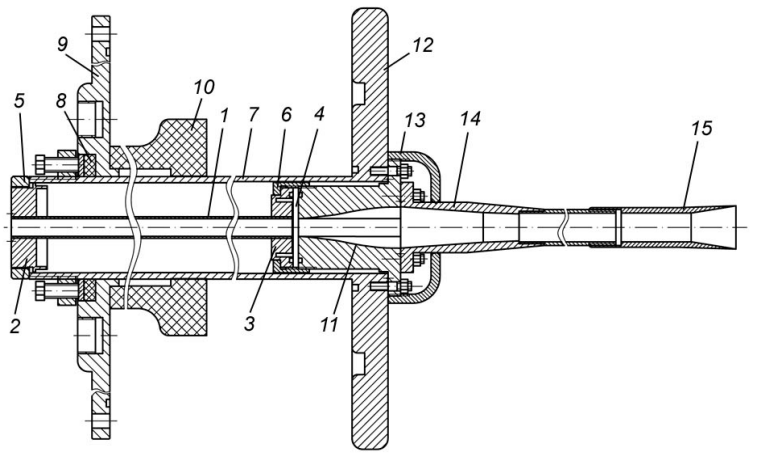

Fig. 2. Microwave transmission system inside the LIA module (schematic).

1 - standard rectangular waveguide; 2,3 - waveguide flanges; 4 - vacuum window; 5,6 - fixing nuts; 7 LIA center electrode; 8 - seal; 9 - end flange of LIA tank; 10 - support for LIA pulsed transformer; 11 buffer section (rectangular-to-circular waveguide transformer); 12 - LIA high-voltage flange; 13 rounded screen; 14 - cathode holder; 15 - cathode.

The design consists of complicated components, and jitters intrinsic to their operation should not prevent the RF drive pulse produced by the compressor from falling at about the middle of the beam (LIA) pulse. The scheme of synchronization has been developed; it is based on the formation of triggering pulses for the LIA, magnetron, and compressor in the single block including artificial delay lines and power amplifiers in each of the three channels and cable delay lines at channels' outputs. Artificial lines are able to set a delay time with the minimum step of $50 \mathrm{~ns}$, and exact synchronization is achieved by adjusting the cable lengths. The necessary condition is that all jitters do not exceed 10-15 ns.

\section{Particle-in-cell simulations}

Simulations of the beam-wave interaction in the device have been carried out using the 3 -D version of PIC code MAGIC. In comparison to our initial work on the antenna-amplifier MAGIC simulation [2] using a simplified geometry and beam emission model, these simulations were performed for the geometry close to the experimental one, including the region of the diode with explosive emission. Both coaxial and quasi-planar geometries of the diode were simulated; no considerable influence on the gain was observed.
The simulation space in case of coaxial diode is depicted in Fig. 3. The dielectric $\operatorname{rod}(\varepsilon=5)$ is inserted inside the conducting cathode. At the left boundary, two ports are set: outer (with respect to the cathode) for the voltage applied, and inner for the microwave input. The right boundary is set as FREESPACE. A diode voltage of $\sim 290 \mathrm{kV}$ and $\sim 1.17 \mathrm{kA}$ beam current were provided. The input signal was injected in the $\mathrm{TE}_{11}$ mode of the waveguide filled with the dielectric. The beam was guided by a $3.0 \mathrm{~T}$ magnetic field.

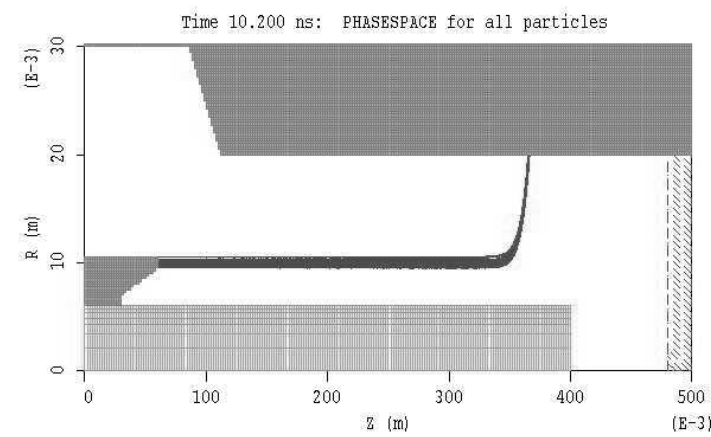

Fig. 3. System geometry and beam axial cross-section in MAGIC simulations of the antenna-amplifier.
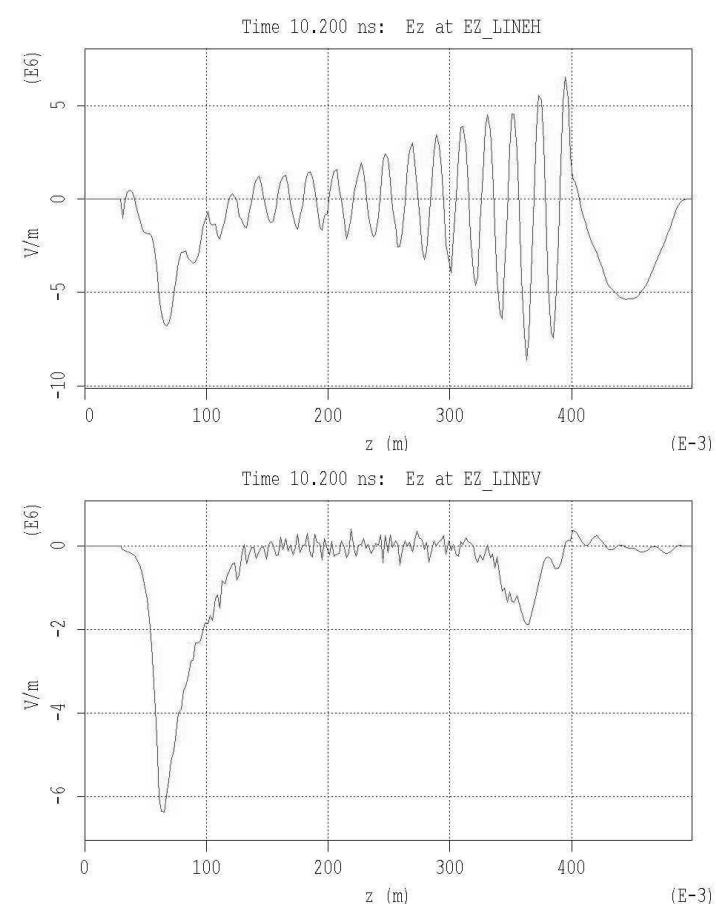

Fig. 4. $E_{z}$ field instantaneous profiles at the rod surface for the azimuths corresponding to the input $\mathrm{TE}_{11}$ mode polarization (top) and displaced by $90^{\circ}$ (bottom). The input power is $\sim 210 \mathrm{~kW}$, the frequency is $9.3 \mathrm{GHz}$.

The achievable gain, bandwidth, and efficiency of the antenna-amplifier were investigated at different values of the input RF power and interaction region length. The peak gain occurs at a $9.3 \mathrm{GHz}$ drive frequency; it is illustrated in Fig. 4, where the instantaneous profiles of axial electric field at the rod surface are presented. The results for gain and bandwidth are 
in the good agreement with those of the linear theory, in particular, some asymmetry of the bandwidth was observed in simulations. At increased interaction region length and input RF power, nonlinear effects are clearly manifested. For a beam length of $30 \mathrm{~cm}$ (counting from the entrance into the drift tube) and $\sim 530 \mathrm{~kW}$ input power, the device efficiency reaches $\sim 15 \%$ at $30-32$ MW output power (observation plane was $1 \mathrm{~cm}$ before the rod end). In the simulation with the finite RF drive pulse length (about twice less than the simulation time), the output power of 20-22 MW was observed.

\section{Experiments on beam transport}

The model experiments on beam transport in the absence of an external microwave signal were supposed to clarify whether plasma formation at the surface of the dielectric rod is admissible for the device operation. Geometry of the experiments is shown in Fig. 5. It includes the LIA vacuum chamber (with its insulator, high-voltage flange, and cathode holder), coaxial magnetically insulated diode, tapered buffer section, and drift tube. Dielectric rods were inserted through the hollow cathode into the hollow end section of cathode holder. The distance from the cathode edge to the rod end was $270 \mathrm{~mm}$ maximum. Rods of three different materials were tested: plexiglass, polyethylene, and quartz.

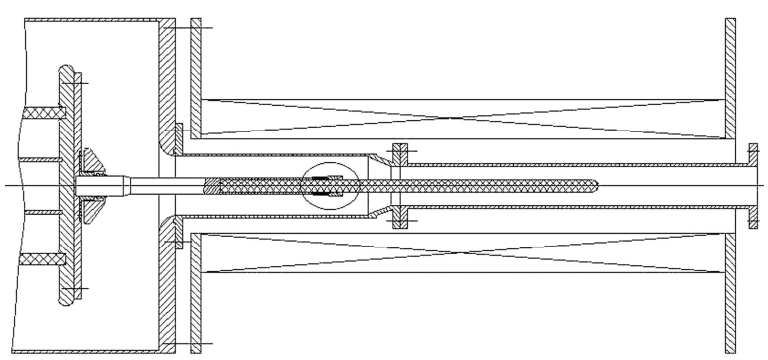

Fig. 5. Schematic of experimental geometry. Circle marks the cathode of the magnetically insulated diode.

The main diagnostic means implemented for estimations of the plasma density near the rod was based on measuring the current transferred by the inside of annular beam. Considering it as the electron saturation current, one can estimate the near-surface plasma electron density. For measurements, the special composite Faraday cup with two collectors was placed downstream the rod end. The annular beam was delivered to its outer collector, and the current inside the beam was delivered to the inner collector through the foil diaphragm. Obtaining dependences of the current delivered to the inner collector on the diaphragm diameter allowed for estimating the plasma density radial profile.

It should be noted that after many shots made in the course of experiments, the plexiglass and polyethylene rods exhibited traces of surface breakdown along all their length from the buffer section to the rod end. In addition, for the plexiglass rod, the small "waist", i.e.,
$0.3 \mathrm{~mm}$ reduction of rod diameter under the cathode edge, has been observed. It is caused by the UV radiation from cathode plasma heating the rod up to material transfer off. For the quartz rods, neither breakdown traces, nor waist were observed. Evidently, plastic rods are rather not appropriate for an operating device, and a material with high thermal strength like quartz, titanium silicate glass, or ceramics should be employed.

With the quartz rods, the series of experiments were performed, and the current delivered to the Faraday cup inner collector was measured at different accelerating voltages, values of guiding magnetic field, and distances between the annular beam and rod surface (the cathode edge diameter was fixed, and varied was the rod diameter). The data were statistically processed to obtain estimations of current density profiles. It has been found that the near-surface current density considerably increases with decreasing guide magnetic field and increasing LIA voltage. Also, it is higher for a smaller beam-rod gap. Nevertheless, for $\sim 280 \mathrm{kV}$ voltage, $\sim 4 \mathrm{~mm}$ gap, and $\sim 2.6 \mathrm{~T}$ guide field, it is $20-40$ $\mathrm{A} / \mathrm{cm}^{2}$ that corresponds to the plasma density of $\sim 10^{12}$ $\mathrm{cm}^{-3}$, rather acceptable for an X-band device. Further investigations are needed with improved vacuum conditions.

\section{Low-power "cold" microwave measurements}

Studies of microwave transmission and antenna pattern without electron beam generation have been carried out using the low-power $(\sim 0.1 \mathrm{~W})$ frequency tunable (7.6-9.8 $\mathrm{GHz}$ ) cw oscillator. Two model polyethylene antennas (of 17 and $25 \mathrm{~cm}$ length) were tested. For comparison, the measurements with the developed microwave transmission system have been made in the anechoic chamber (case of isolated antenna) and with the system incorporated into the LIA chamber assembled with the electron diode and drift tube used in experiments on beam transport, i.e., in the real geometry of antenna-amplifier device, except for a vacuum window presence.

It has been found that the real geometry of the device practically does not change the angular distribution of radiated power and its dependence on frequency. Measurements have shown that the longer antenna has a higher directivity as expected ( $\sim 15-17^{\circ}$ FWHM angular width), however, it exhibits much faster growth of the side lobes level with increasing frequency. Also, it has been shown that the reflected signal can be eliminated under conditions of real surrounding of the dielectric rod. This is important in the light of future experiments on gain demonstration in the antenna-amplifier.

\section{References}

[1] A.S.Shlapakovski, in "Intense Microwave Pulses VI", SPIE Proc., Volume 3702, 1999, pp.108-113.

[2] A.S.Shlapakovski, I.I.Vintizenko, A.V.Petrov, and E.Schamiloglu, in Proc. $13^{\text {th }}$ Symposium on HighCurrent Electronics, 2004, pp. 224-227. 